2001

\title{
Inhomogeneous Transverse Electric Fields And Wave Generation In The Auroral Region: A Statistical Study
}

Maria Hamrin

Mats André

Gurudas Ganguli

Valeri V. Gavrishchaka

Mark E. Koepke

See next page for additional authors

Follow this and additional works at: https://researchrepository.wvu.edu/faculty_publications

\section{Digital Commons Citation}

Hamrin, Maria; André, Mats; Ganguli, Gurudas; Gavrishchaka, Valeri V.; Koepke, Mark E.; Zintl, Michael W.; Ivchenko, Nickolay; Karlsson, Tomas; and Clemmons, James H., "Inhomogeneous Transverse Electric Fields And Wave Generation In The Auroral Region: A Statistical Study" (2001). Faculty Scholarship. 564.

https://researchrepository.wvu.edu/faculty_publications/564 


\section{Authors}

Maria Hamrin, Mats André, Gurudas Ganguli, Valeri V. Gavrishchaka, Mark E. Koepke, Michael W. Zintl, Nickolay Ivchenko, Tomas Karlsson, and James H. Clemmons 


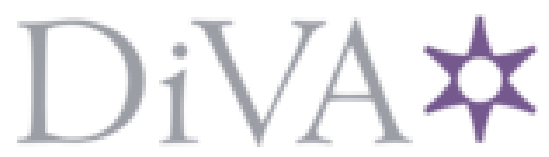

http://www.diva-portal.org

This is the published version of a paper published in Journal of Geophysical Research.

Citation for the original published paper (version of record):

Hamrin, M., Andre, M., Ganguli, G., Gavrishchaka, V., Koepke, M. et al. (2001)

Inhomogeneous transverse electric fields and wave generation in the auroral region: A statistical study.

Journal of Geophysical Research, 106(A6): 10803-10816

http://dx.doi.org/10.1029/2001JA900003

Access to the published version may require subscription.

N.B. When citing this work, cite the original published paper.

Permanent link to this version:

http://urn.kb.se/resolve?urn=urn:nbn:se:umu:diva-85977 


\title{
Inhomogeneous transverse electric fields and wave generation in the auroral region: A statistical study
}

\author{
Maria Hamrin, ${ }^{1}$ Mats André, ${ }^{2}$ Gurudas Ganguli, ${ }^{3}$ Valeri V. Gavrishchaka, ${ }^{4}$ \\ Mark E. Koepke ${ }^{5}$ Michael W. Zintl ${ }^{5}$ Nickolay Ivchenko, ${ }^{6}$ Tomas Karlsson, ${ }^{6}$ \\ and James H. Clemmons ${ }^{7}$
}

\begin{abstract}
We use data from the Freja satellite to investigate the importance of localized transverse DC electric fields for the generation of broadband waves responsible for ion heating in the auroral region. Theoretical models indicate that shear in the plasma flow perpendicular to the geomagnetic field can generate waves in a broad range around the ion gyrofrequency for parallel currents significantly below the threshold of the current-driven electrostatic ion cyclotron instability. We compare in situ data with laboratory measurements and theoretical predictions, and we find that inhomogeneous electric fields might well be important for the generation of waves in the auroral region.
\end{abstract}

\section{Introduction}

Understanding and predicting the magnetospheric observations of ion energization using theoretical models is an important and intriguing problem in space physics. Ions from the ionosphere are observed to be heated perpendicularly to the divergent geomagnetic field, and the outflow of these ions, caused by their increased magnetic moment, supplies the upper magnetosphere with a significant fraction of its plasma. At least at altitudes from $1000 \mathrm{~km}$ up to several thousand kilometers, most of this ion energization is associated with broadband waves. These waves (sometimes called broadband extremely low frequency waves (BB-ELF) [Knudsen et $a l ., 1998])$ are electric and magnetic field fluctuations observed in the range $1 \mathrm{~Hz}-3 \mathrm{kHz}$, and they are often found in the auroral region. Gyroresonant heating by waves around the ion gyrofrequency [Lysak et al., 1980; Dusenbery and Lyons, 1981; Chang et al., 1986] makes a plausible ion energization mechanism [Norqvist et al., 1996, 1998; Moore et al., 1999; Hultqvist et al., 1999],

\footnotetext{
${ }^{1}$ Theoretical Space Physics, Umeå, Sweden.

${ }^{2}$ Uppsala Division, Swedish Institute of Space Physics, Uppsala, Sweden.

${ }^{3}$ Plasma Physics Division, Naval Research Laboratory, Washington, D. C.

${ }^{4}$ Science Applications International Corporation, McLean, Virginia.

${ }^{5}$ Physics Department, West Virginia University, Morgantown, West Virginia.

${ }^{6}$ Royal Institute of Technology, Stockholm, Sweden.

${ }^{7}$ The Aerospace Corporation, El Segundo, California.
}

Copyright 2001 by the American Geophysical Union.

Paper number 2001JA900003.

0148-0227/01/2001JA900003\$09.00 but specifics of the wave generation mechanism are still being debated.

The current-driven electrostatic ion cyclotron (CDEIC) instability [Drummond and Rosenbluth, 1962; Kindel and Kennel, 1971] has been invoked in the past to explain the origin of these broadband waves. Recently, good correlation has been reported between broadband waves, ion heating, and the presence of magneticfield-aligned (i.e. parallel) currents in the magnetosphere [Bonnell et al., 1996; Kintner et al., 1996; André et al., 1998]. However, explaining this correlation by an ion-heating mechanism based on the CDEIC instability brings about fundamental problems. For example, estimates made from in situ data of the parallel electron drift speed responsible for the parallel current are typically far below the excitation threshold of the CDEIC instability. Moreover, since the CDEIC wave excitation threshold increases with increasing ion temperature, there is a self limitation in the wave growth and consequently in the ability of CDEIC waves to heat ions [Palmadesso et al., 1974]. It is also found that broadband wave spectra observed in space lack the narrow cyclotron harmonic features expected over a wide range of parameters from waves generated by the CDEIC instability.

These discrepancies suggest that the CDEIC instability is alone inadequate as a mechanism for generating the broadband waves needed to energize the ionospheric ions. In this article we evaluate a mechanism capable of destabilizing waves (1) at currents significantly below the CDEIC excitation threshold, (2) with significantly less sensitivity to increases of ion temperature, (3) with a spectrum significantly broader than the CDEIC wave spectral feature, and (4) at frequencies shifted to either side of the ion gyrofrequency. This mechanism depends on a localized transverse DC electric field [Ganguli et 


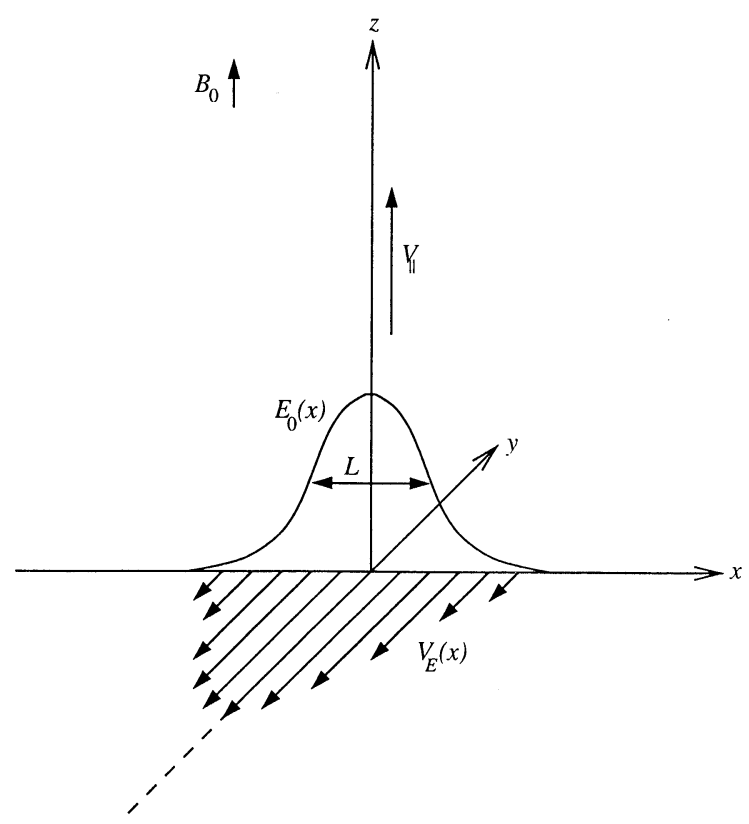

Figure 1. Simplified equilibrium configuration of the model plasma. There is a localized transverse electric field $E_{0}(x)$ in the $x$ direction causing a sheared plasma flow $V_{E}(x)$ in the negative $y$ direction. The DC electric field profile is assumed to have a Gaussian shape with the scale length $L$. The background magnetic field $B_{0}$ is along the $z$ axis, and there is a uniform field-aligned drift $V_{\|}$of the electrons relative to the ions.

al., 1985a]. In its simplest form it was shown by Ganguli et al. $[1985 \mathrm{a}, 1988]$ that a localized transverse DC electric field could lead to a localized region of negative wave energy density. When this region is coupled to a region of positive wave energy density, this can result in a nonresonant instability for frequencies around the ion gyrofrequency. This instability grows at the expense of the electric field inhomogeneity and does not depend on a field-aligned current and the resulting waves can be an efficient ion heating source [Ganguli et al., 1985b]. Since the instability is attributed to an inhomogeneity in the energy density, it has been referred to as the inhomogeneous energy density-driven (IEDD) instability. Following this, Ganguli and Palmadesso [1988] studied the combined effects of transverse localized electric fields and a field-aligned current. They found that a DC electric field could change the resonance properties and lead to ion cyclotron instability with a substantially lower current threshold as compared to the classical CDEIC instability. Subsequently, a number of detailed studies have characterized this resonant instability, which derives its free energy from a field-aligned electron drift that is subcritical to the classical CDEIC instability [Ganguli et al., 1994; Gavrishchaka et al., 1996]. More recently, a number of laboratory experiments were designed to validate the mechanism and test the ion heating capabilities of both the resonant and also the nonresonant waves [Amatucci et al., 1994, 1996; Koepke and Amatucci, 1992; Koepke et al., 1994; Carroll et al., 1998; Walker et al., 1997].
Most naturally occurring plasmas are inhomogeneous, and it is widely known that plasma inhomogeneities can give rise to wave instabilities. Among the first studies, Mozer et al. [1977] and Kelley and Carlson [1977] surmised that localized electric fields in the ionosphere and the magnetosphere could be important for the generation of waves. As discussed above, a decade later it was shown that waves in a broad range around the ion gyrofrequency could be destabilized by the combination of field-aligned currents and localized electric fields. Another decade later, nonuniform parallel flows were predicted [Gavrishchaka et al., 1999, 2000] to drive waves within a broad frequency range from much below the ion gyrofrequency to much above it, but in this article we will focus only on inhomogeneities in the transverse plasma flows.

Attempts to compare the theory of localized transverse electric fields with in situ measurements from satellites and sounding rockets in the magnetosphere have been made [Basu et al., 1988; Earle et al., 1989; Moore et al., 1996; Bonnell et al., 1996; Bonnell, 1997; Kivanç and Heelis, 1999; Kintner et al., 1996, 2000]. These investigations all point to the importance of localized transverse fields in space for the generation of waves. However, a statistical investigation of these localized electric fields to evaluate their significance for the generation of the waves responsible for the ion energization of the ionospheric ions has never been done. The statistical study we present here correlates localized perpendicular electric fields, parallel currents, and broadband waves. Rather than just correlating localized electric fields with waves around the oxygen gyrofrequency, i.e., essentially concluding that the waves are broadbanded, we test if the observed electric field strengths are large enough to cause waves at higher frequencies by comparing with theory and laboratory data. However, because of the space-time ambiguity in the Freja data, we use certain assumptions in our analysis of the velocity shear.

It has recently been suggested that significant spatial electric field structures, at least sometimes, are present during ion heating events and also that some of the observed broadband wave structures can be due to Doppler shifts from the satellite motion [Stasiewicz et al., 2000]. It has also been suggested that the ions could be heated directly by nonadiabatic motion in electrostatic structures alone without the need for energizing broadband waves [Mishin and Banaszkiewicz, 1998]. In this article we will, however, not focus on these suggestions.

We find that gradients in the localized electric field might well be important for the generation of broadband waves in the magnetosphere. We here note that the waves we discuss are not related to the solitary structures (electron phase space holes) sometimes observed in the auroral region [Ergun et al., 1998]. These structures may also heat ions, and the solitary structures in real space give broadband structures in frequency. Section 2 briefly reviews the theoretical model 


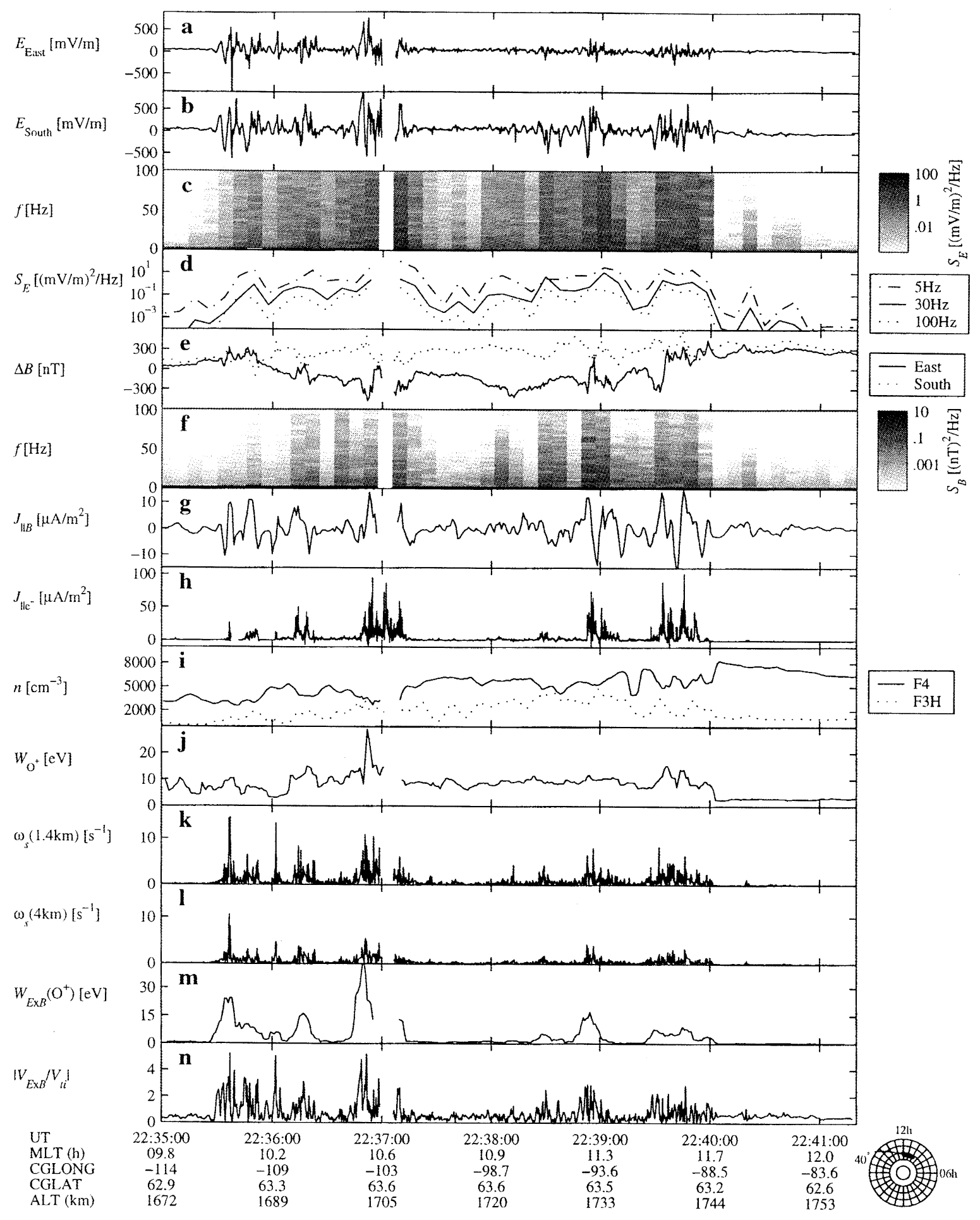

Figure 2. Data from Freja orbit 6656. Time series of (a) eastward electric field component; (b) southward electric field component; (c) electric field power spectral density; (d) electric field power spectral density at certain frequencies; (e) magnetic field variations; (f) magnetic field power spectral density; ( $\mathrm{g}$ ) field-aligned magnetometer current; (h) net current from high-energy electrons; (i) plasma density from the F4 (Langmuir probe) and the F3H (mass spectrometer) instrument, respectively; (j) perpendicular $\mathrm{O}^{+}$ion energy; $(\mathrm{k})$ shear frequency; (l) shear frequency; $(\mathrm{m})$ energy of $\mathbf{E} \times \mathbf{B}$ drifting $\mathrm{O}^{+}$ions, and $(\mathrm{n}) \mathbf{E} \times \mathbf{B}$ drift normalized to the $\mathrm{O}^{+}$ion thermal speed. Structures of scale lengths longer than $1.4 \mathrm{~km}$ in Figures $2 \mathrm{k}$ and $2 \mathrm{~m}$ and $4 \mathrm{~km}$ in Figure $2 \mathrm{l}$ are assumed static. There is a clear correlation of wave activity with large and highly varying shear frequencies and also with field-aligned currents. 
used for our predictions, and section 3 contains a detailed study of the field and particle data from an event in the dayside magnetosphere. In section 4 we perform a statistical analysis of this event and of a second event from the nightside magnetosphere. We also compare the statistical results with theoretical predictions and laboratory measurements. The two last sections contain a discussion and the conclusions of our overall results.

\section{Inhomogeneous Plasmas}

Inhomogeneities in plasma parameters such as the density and drifts are inescapable in many plasmas, both in the designed conditions of a laboratory experiment and in the expanse of a space plasma. Such inhomogeneities can naturally cause wave instabilities.

We use a simplified equilibrium configuration of the model plasma, shown in Figure 1, with a uniform plasma density $n$, magnetic field $B_{0}$ in the $z$ direction, and field-aligned electron drift (relative to the ions) $V_{\|}$. In general, both the parallel electron drift and the plasma density could also be nonuniform, but in the following we will disregard this fact. There is a perpendicular DC electric field $E_{0}(x)$ in the $x$ direction, the magnitude of which varies along the $x$ axis. This field causes a sheared flow of $\mathbf{E} \times \mathbf{B}$ drifting plasma in the negative $y$ direction as indicated in the figure. This plasma flow profile $V_{E}(x)$ is characterized by the full width at half maximum $L$. Waves in a broad frequency range around the ion gyrofrequency can be destabilized for quite small amounts of such sheared transverse flows. Depending on the wave modes generated, the instability requires no current or significantly less current than the CDEIC instability.

Ganguli et al. [1988] derived a general eigenvalue condition for the basic setup in Figure 1 and described the properties of the inhomogeneous energy density-driven instability. These IEDD waves are distinct from CDEIC waves. For example, the IEDD wave frequency can be shifted by approximately $k_{y} V_{E}$ from the CDEIC wave frequency. For one class of waves the wave generation does not depend on a field-aligned current. These nonresonant waves dissipate the electric field as they grow. Another class of waves can be excited by localized electric fields in conjunction with some field-aligned current [Ganguli and Palmadesso, 1988]. These are resonant waves that feed on the field-aligned current similar to CDEIC waves, but they can grow where the CDEIC waves are stable. It has also been found that these waves are not as sensitive as the classical CDEIC waves to the ion-to-electron temperature ratio $T_{i} / T_{e}$. In this article we assume that the observed electric field can be approximated by a Gaussian profile $E_{0}(x)$ with a full width at half maximum $L$. This allows us to approximate the maximum gradient of $V_{E}(x)$ as the maximum of $V_{E}(x)$ divided by $L$. Theoretical studies using different velocity profiles have shown that the wave characteristics are not very sensitive to the sharpness of the velocity profile [Ganguli et al., 1988; Gavrishchaka et al., 1996].

In the theoretical model discussed above, the growth rate of the waves is a function of the peak value $V_{0 E}$ of the $\mathbf{E} \times \mathbf{B}$ drift and the scale size $L$ of the velocity profile, but not necessarily of the joint parameter $V_{0 E} / L$. However, to be able to compare theoretical predictions, laboratory results, and data acquired by a satellite or a sounding rocket, it is useful to introduce a scaling quantity called the shear frequency that combines the quantities $L$ and $V_{0 E}$ according to

$$
\omega_{s}=\left|\frac{d V_{E}(x)}{d x}\right|_{\max } \approx \frac{V_{0 E}}{L},
$$

where $V_{E}(x)$ is the $\mathbf{E} \times \mathbf{B}$ drift. This is a global parameter characterizing the entire flow profile as opposed to the local shear, $d V_{E}(x) / d x$, which is different at different positions within the flow profile. The theoretical model requires $\omega_{s}$ to be less than the ion gyrofrequency $\Omega_{i}$ and $L>\rho_{i}$, where $\rho_{i}$ is the ion gyroradius. The first condition is easily met in plasmas in the auroral region, whereas meeting the second condition is uncertain owing to the space-time ambiguity in the in situ data. However, our assumption used in interpreting the data is consistent with $L>\rho_{i}$.

\section{Observations}

From the joint Swedish and German satellite Freja we present data to investigate the possible influence of sheared transverse plasma flows and field-aligned currents on the generation of waves at frequencies around the oxygen gyrofrequency in the magnetosphere. Freja passes the northern auroral region at an altitude of $\sim 1700 \mathrm{~km}$ and at an inclination of $\sim 63^{\circ}$. Its lowinclination orbit gives a rather unique perspective of auroral phenomena, since it, at times, moves along the auroral oval instead of across it, as for polar-orbiting satellites. An overview of the Freja instrumentation is given by Lundin et al. [1994, and references therein]. In this article we use electric field data from the F1 instrument and magnetic field data from the F2 instrument. Also wave and density data from the F4 instrument, ion data from the $\mathrm{F} 3 \mathrm{H}$ instrument, and electron data from the F7 instrument are used.

In Figure 2 we show electric and magnetic field and particle data from Freja orbit 6656 on February 21, 1994. This event includes $\sim 6$ min of data from the dayside magnetosphere. Precipitating $\mathrm{H}^{+}$ions, electrons accelerated to energies of several hundreds $\mathrm{eV}$, and broadband waves (but almost no lower hybrid waves) are present during the event [see Norqvist et al., 1996, Figure 1]. Note that at about 2237:00 UT there is a data gap because of a shift of satellite antennas.

Figures $2 \mathrm{a}$ and $2 \mathrm{~b}$ show two components of the electric field (F1) perpendicular to a model geomagnetic field. The components are directed toward magnetic east and magnetic south, respectively. The electric field 
is measured in the spin plane of Freja, and for the coordinate transformation the third component, which completes the right-handed orthogonal set, is assumed to be zero. We have filtered the data by calculating overlapping running averages, so that electric field variations of $0.2 \mathrm{~s}$ and shorter measured by Freja are removed. With a satellite velocity of approximately $V_{\text {sat }} \approx 6.8$ $\mathrm{km} / \mathrm{s}$ perpendicular to the background magnetic field, we will assume that all static DC electric field structures have a scale size $1.4 \mathrm{~km}$ and longer.

In Figures $2 \mathrm{a}$ and $2 \mathrm{~b}$ we see that the electric field occasionally is quite large, reaching values of a few hundred $\mathrm{mV} / \mathrm{m}$. We also see that the field varies rapidly and unevenly, indicating that the flow and its shear are inhomogeneous.

Figure 2c shows the power spectral density for frequencies up to $100 \mathrm{~Hz}$ of an electric field component measured with two probes separated by $21 \mathrm{~m}$ in the spin plane of Freja. Data from the F4 instrument is used, and the spectral density is estimated from 1.5-s-long time series sampled every $8 \mathrm{~s}$. When comparing these data with Figures $2 \mathrm{a}$ and $2 \mathrm{~b}$, we see that the spectral density often is relatively broadband and usually coinciding with intervals of highly varying, large electric fields. This is also evident from Figure 2d, where the power spectral densities at the frequencies 5,30 , and $100 \mathrm{~Hz}$, respectively, are plotted.

Figure 2e contains the geomagnetic east and south components of the magnetic field (F2) with a model field subtracted. Similarly to the electric field data, variations in the magnetometer data shorter than 0.5 $\mathrm{s}$ are removed by calculating overlapping running averages. Figure $2 \mathrm{f}$ contains a plot of the F4 power spectral density of the magnetic wave data produced in the same way as the electric field spectral density in Figure 2c. We see that when the electric field in Figures $2 a$ and $2 b$ is large and highly varying, the power spectral densities of the magnetic field (Figure 2f) and also of the electric field (Figure 2c) is high and relatively broadbanded.

From the magnetic field data, the field-aligned current is estimated by means of a method described by Lühr et al. [1996]. The parallel current in Figure $2 \mathrm{~g}$ is filtered by means of running averages so that variations faster than $3 \mathrm{~s}$ (i.e., half a satellite spin) are removed, and therefore small-scale variations of the current are not shown.

Figure $2 \mathrm{~h}$ contains F7 electron spectrometer data of the contribution from energetic electrons $(\sim 20 \mathrm{eV}$ to 25 $\mathrm{keV}$ [Boehm et al., 1994]) to the field-aligned current. This contribution is measured with much higher resolution than the magnetometer current in Figure 2g. The observations of electrons at energies above $\sim 20 \mathrm{eV}$ indicate that structures of shorter scale lengths are present, and possibly such structures could also be present at lower energies.

The plasma density from a F4 Langmuir probe averaged over one satellite spin is shown as a solid line in Figure 2i. The dotted line indicates the $\mathrm{O}^{+}$density ob- tained from the $\mathrm{F} 3 \mathrm{H}$ ion mass spectrometer averaged in the same way (see Waldemark and Norqvist [1995] for details concerning the $\mathrm{F} 3 \mathrm{H}$ calibration). By comparing the two density estimates, we find that at least when the wave activity is high, a significant fraction of the ions are $\mathrm{O}^{+}$and they have an energy of at least a few $\mathrm{eV}$ (a limit set by the satellite velocity as discussed in the next paragraph).

Figure $2 \mathrm{j}$ shows the average transverse $\mathrm{O}^{+}$ion energy obtained from the $\mathrm{F} 3 \mathrm{H}$ instrument, averaged over one satellite spin. The minimum energy the $\mathrm{O}^{+}$ions can have in the satellite frame (rammed ions) is determined by the satellite velocity. This velocity of $\sim 7 \mathrm{~km} / \mathrm{s}$ corresponds to an $\mathrm{O}^{+}$energy of $\sim 4 \mathrm{eV}$. After $2240 \mathrm{UT}$ the $\mathrm{O}^{+}$energy is close to this limit, and therefore the density estimates from this instrument are not meaningful (Figure 2i). Together, Figures $2 \mathrm{i}$ and $2 \mathrm{j}$ show that when the wave activity is high, a significant fraction of the $\mathrm{O}^{+}$ ions are heated to average energies of $10-20 \mathrm{eV}$.

Figures $2 \mathrm{k}$ and $2 \mathrm{l}$ show the estimated local value of the shear frequency defined as the spatial derivative along the satellite orbit of the orthogonal component of the $\mathbf{E} \times \mathbf{B}$ drift. However, the data in Figure 2 cannot be used to distinguish between electric field variations in time and space. In the following discussion we will make the important assumption that field structures at long enough scale lengths are static. We assume that time variations of duration $T$ and longer are due to the satellite moving at a velocity $V_{\text {sat }} \approx 6.8 \mathrm{~km} / \mathrm{s}$ (perpendicular to the background magnetic field) through static structures. This means that time-varying fields observed in the satellite frame at frequencies below $1 / T$ $\mathrm{Hz}$ are caused by Doppler shift owing to the spacecraft motion through structures longer than $V_{\text {sat }} T$. Since we study the generation of waves around $26 \mathrm{~Hz}$, i.e., the $\mathrm{O}^{+}$ gyrofrequency at the Freja altitude, we want to separate in the frequency domain (as observed by the satellite) the static structures possibly causing the broadband waves from the broadband waves themselves. In Figure $2 \mathrm{k}$ we estimate the shear by using $T=0.2 \mathrm{~s}$, corresponding to a Doppler-shifted frequency of $5 \mathrm{~Hz}$, which is well below $26 \mathrm{~Hz}$. This $T$ corresponds to static structures of $1.4 \mathrm{~km}$ or longer, which for $\mathrm{O}^{+}$ions with energies of $10 \mathrm{eV}$ is approximately equal to 29 ion gyro radii, $\rho_{i}$. In the same way, in Figure 21 we use 0.6 $\mathrm{s}\left(1.6 \mathrm{~Hz}, 4 \mathrm{~km}, 88 \rho_{i}\right)$. Comparing the two panels, we find that the shear frequency decreases as the assumed $T$ and the corresponding length scale increases, as expected from (1). Also, both panels together with Figures $2 \mathrm{a}-2 \mathrm{f}$ show a good correlation between high estimated shear frequency and broadband waves.

In principle, the polarization of the observed electric and magnetic fields can be used to distinguish between waves and static structures by using only a single spacecraft. For example, the ratio $|\mathbf{E}| /|\mathbf{B}|$ should be equal to the ionospheric conductivity for some static structures, or the Alfvén speed in the case of some Alfvén waves. In reality, the analysis is complicated 


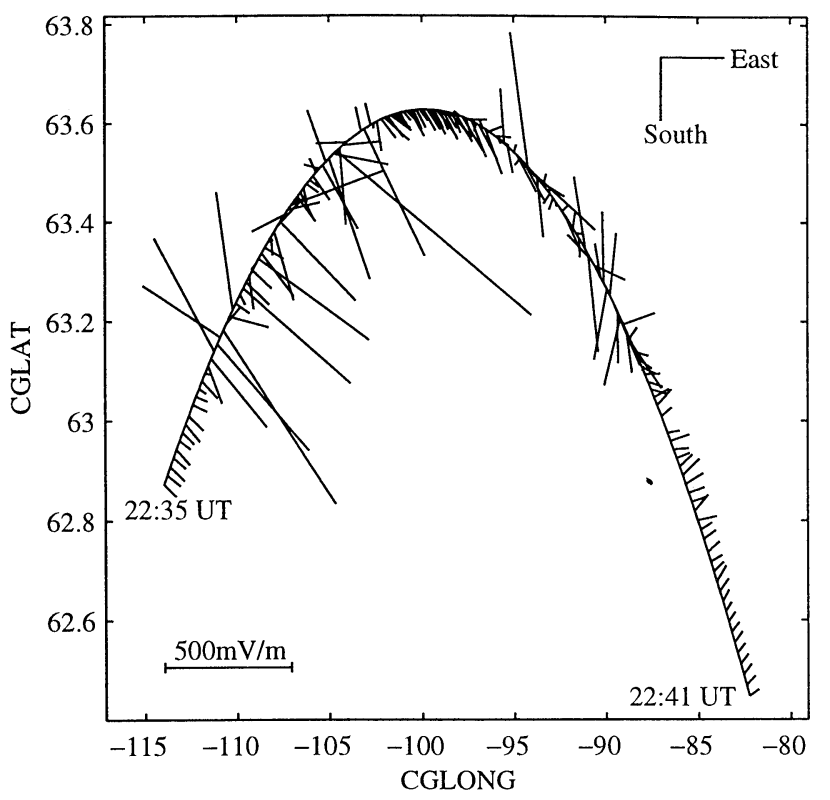

Figure 3. Electric field vectors perpendicular to the geomagnetic field and along the satellite orbit for the dayside event. Magnetic east is to the right, and magnetic south is downward. The vectors are plotted at intervals of $2 \mathrm{~s}$, and their scaling is indicated at bottom left. The electric field is highly varying during the event.

by several factors, including the possible presence of static electric fields parallel to the geomagnetic field, a mixture of downgoing and upgoing Alfvén waves (the latter being reflected at the ionosphere), and Alfvén waves spread over a wide range of wavelengths (where the wavelengths will influence the polarization). Previous studies of low-frequency waves include investigations of data from the DE 1 satellite [Gurnett et al., 1984], the Intercosmos-Bulgaria-1300 spacecraft [ $\mathrm{Du}$ binin et al., 1985], the Aureol-3 satellite [Berthelier et al., 1991], data from a combination of ground-based magnetometer and European Incoherent Scatter (EISCAT) radar observations together with Viking spacecraft data [Aikio et al., 1996], and data from the Freja satellite [Stasiewicz et al., 2000]. The presence of a mixture of phenomena is often concluded. The study by Stasiewicz et al. [2000] suggests that, sometimes, linear Alfvén waves exist over a wide range of wavelengths, obeying a wave dispersion relation but maybe at such a low frequency that they, as compared to the ion motion, can be regarded as static structures. This interpretation might be ambiguous [see, e.g., Wahlund et al., 1998], but it shows how complex the real situation is. In our present study we simply assume that all electric fields observed by Freja at low frequencies are due to Doppler shifts by the satellite motion of static electric field structures. Our goal is to test if it is feasible that gradients in such structures can be important for the generation of waves near the ion gyrofrequency or if such an hypothesis can be disregarded.
The energy of the oxygen ions associated with the $\mathbf{E} \times \mathbf{B}$ drift, and calculated by using the electric field averaged over $1.4 \mathrm{~km}$ as in Figures $2 \mathrm{a}$ and $2 \mathrm{~b}$, is shown in Figure $2 \mathrm{~m}$. In Figure $2 \mathrm{n}$ the $\mathbf{E} \times \mathbf{B}$ drift velocity normalized to the $\mathrm{O}^{+}$ion thermal velocity (obtained from Figure $2 \mathrm{j}$ ) is presented. Sometimes the energy corresponding to the $\mathbf{E} \times \mathbf{B}$ drift is equal to or somewhat larger than the average $\mathrm{O}^{+}$energy in Figure $2 \mathrm{j}$. Note that the transverse electric field here causes a perpendicular drift, rather than bulk heating of the ion distribution to some higher temperature. If our assumptions concerning the scale size of the static electric fields are correct, then sometimes no broadband waves are needed to energize the ions to the observed average energies. However, detailed observations of the distribution functions show significant bulk heating during this event [see Norqvist et al., 1996, Figures 2 and 3] and hence energizing mechanisms other than the $\mathbf{E} \times \mathbf{B}$ drift are needed.

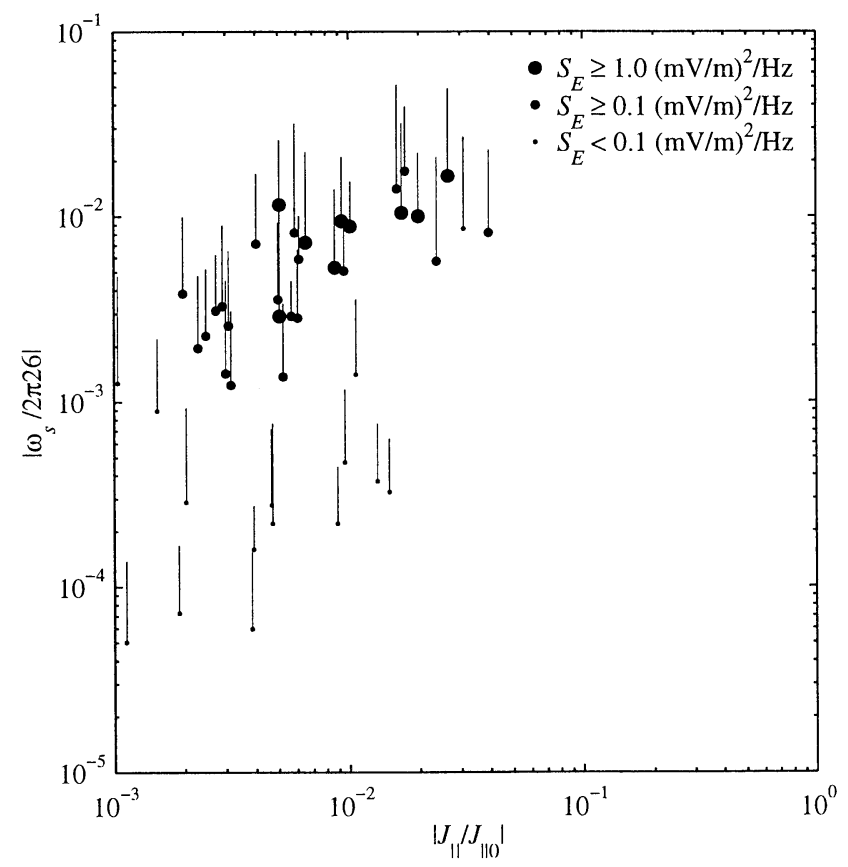

Figure 4. Normalized shear frequency plotted versus the absolute value of the normalized parallel magnetometer current for the dayside event. The data are sorted by the power spectral density around the oxygen gyrofrequency $26 \mathrm{~Hz}$ as indicated by solid circles of different sizes. The assumption that electric field structures of $1.4-\mathrm{km}$ size ( $29 \rho_{i}$ of $10 \mathrm{eV}$ oxygen ions) or larger are static is used in the calculation of the shear frequency. Each solid circle marks the average shear frequency calculated from $1.5 \mathrm{~s}$ of data, and the lines indicate the maximum value reached during the time interval. The estimated parallel current is well below the current-driven electrostatic ion cyclotron (CDEIC) critical current $J_{\| 0}$. Higher values of the wave activity reside in the upper part of the figure, indicating the importance of transverse electric fields and sheared flows for the generation of the waves. 
In Figure 3 the electric field perpendicular to the geomagnetic field from Figures $2 \mathrm{a}$ and $2 \mathrm{~b}$ is plotted along the satellite orbit. For clarity, the electric field is plotted only once every $2 \mathrm{~s}$.

To summarize the results from Figures 2 and 3 , a clear correlation between wave activity, ion energization, and large and highly varying electric fields and therefore high shear frequencies can be observed. In the next section we further investigate the importance of high shear frequencies for the generation of waves around the $\mathrm{O}^{+}$gyrofrequency in the magnetosphere.

\section{Statistical Analysis}

In the following we statistically investigate two Freja events, each including several minutes of data. First, we consider the dayside event discussed above, and then we study a nightside event.

\subsection{Event 1: Dayside}

Figure 4 shows the average normalized shear frequency versus the average normalized field-aligned magnetometer current for the dayside event (orbit 6656). The current and shear frequency are obtained from Figures $2 \mathrm{~g}$ and $2 \mathrm{k}$. Also, in Figure 4 the data are sorted by the electric field spectral density $S_{E}$ (as observed in the moving satellite frame) at the $\mathrm{O}^{+}$gyrofrequency around $26 \mathrm{~Hz}$, so that larger circles correspond to higher spectral densities. A large circle indicates that the spectral density around $26 \mathrm{~Hz}$ is high, $S_{E} \geq 1.0(\mathrm{mV})^{2} / \mathrm{m}$, and a circle of intermediate size indicates that the spectral density lies in the interval $0.1(\mathrm{mV})^{2} / \mathrm{m}<S_{E} \leq 1.0(\mathrm{mV})^{2} / \mathrm{m}$. A small circle corresponds to $S_{E} \leq 0.1(\mathrm{mV})^{2} / \mathrm{m}$. We see that higher spectral densities are associated with larger shears.

Since the shear frequency used in Figure 4 is calculated from Figure 2k, it is assumed that structures with a scale length larger than $1.4 \mathrm{~km}$ are static. Both the shear frequency and the current are averaged over the same time interval needed to obtain $S_{E}$, i.e., $1.5 \mathrm{~s}$, which corresponds to $\sim 10 \mathrm{~km}$ along the satellite orbit. Each solid circle corresponding to an average shear frequency in Figure 4 has a line attached to it. The upper end of every line indicates the maximum value of the shear frequency within each 1.5-s interval.

The shear frequency in Figure 4 is normalized to the $\mathrm{O}^{+}$gyrofrequency of $26 \mathrm{~Hz}$. For the normalization of the field-aligned current, we use the critical current $J_{\| 0}$ for the CDEIC instability calculated according to Kindel and Kennel [1971]. They assume that the electrons can be described by a Maxwellian distribution drifting along the magnetic field. In the calculation of $J_{\| 0}$ we assume that all ions are $\mathrm{O}^{+}$and that the electron temperature is $1 \mathrm{eV}$, and we use the $\mathrm{F} 4$ Langmuir probe density in Figure 2i. Furthermore, we assume that the critical parallel electron drift velocity divided by the electron thermal velocity varies linearly with the ion temperature in Figure 2j (see also Figure 2 of Kindel and Kennel [1971]). However, we have neither shown that all electrons are drifting nor checked their temperature. A more reliable way to investigate the possibility of wave generation by field-aligned electrons would be to use high time resolution electron measurements over an appropriate energy range and to study the possibility of various instabilities using kinetic plasma theory; see Gustafsson et al. [1990] for an example involving higher electron energies. However, our approximations indicate that the current most of the time is at least 1 order of magnitude below the threshold for CDEIC wave generation, a tendency which agrees with other studies [Bonnell et al., 1996; André et al., 1998; Kintner et al., 2000]. Thus alternatives to wave generation by field-aligned currents alone must be considered.

From Figure 4 we see that the wave activity increases from the lower part of the figure toward the upper part, so that the wave activity around $26 \mathrm{~Hz}$ is higher when the shear frequency is higher. However, it is less clear that the wave amplitude is related to the current. The reason could be that the parallel current density is far below the critical current density $J_{\| 0}$ of the CDEIC instability and that small variations in the current are not important for the IEDD instability. (However, studies of several events have indicated a correlation between wave activity and $J_{\|}$[André et al., 1998].) There are several data points with $\omega_{s} / \Omega_{i} \sim 10^{-2}, J / J_{\| 0} \sim 10^{-2}$, and moderate or high wave activity. This indicates that a combination of sheared plasma flows and parallel currents could indeed be important for the generation of broadband waves in the magnetosphere. The theoretical model demands that $\omega_{s}<\Omega_{i}$. Note that the validity of the theory hence is assured since $\omega_{s}<\Omega_{i}$ in Figure 4. Also since $J \ll J_{\| 0}$, it is unlikely that the waves are generated solely by the field-aligned current.

From the theoretical models it is predicted that the wave growth depends on the global parameters of the transverse inhomogeneous plasma drift such as the scale length $L$ and the peak value $V_{0 E}$ of the transverse drift. Therefore a global parameter such as the one defined in (1) could be useful when simplifying the comparison with theory. However, for practical reasons when analyzing our in situ data, we characterize the plasma profile with the local value of the shear frequency as the spatial derivative of the $\mathbf{E} \times \mathbf{B}$ drift along the satellite orbit. The points in Figure 4 (average values of the shear frequency) correspond to lower limits of the shear frequency of interest for the wave generation, while the lines (maximum values) give a higher value of this shear. The assumptions of relatively large scale sizes $(1.4 \mathrm{~km})$ of the static electric field structures also imply that the shear frequency is underestimated (see section 4.3). Moreover, the fact that the Freja satellite most likely moves at an angle to the sheared plasma flows implies a further underestimate of the shear frequency, since only the component of the $\mathbf{E} \times \mathbf{B}$ drift orthogonal to the satellite orbit can be differentiated. In spite of some quantitative uncertainty in the estimated $\omega_{s}$ and 


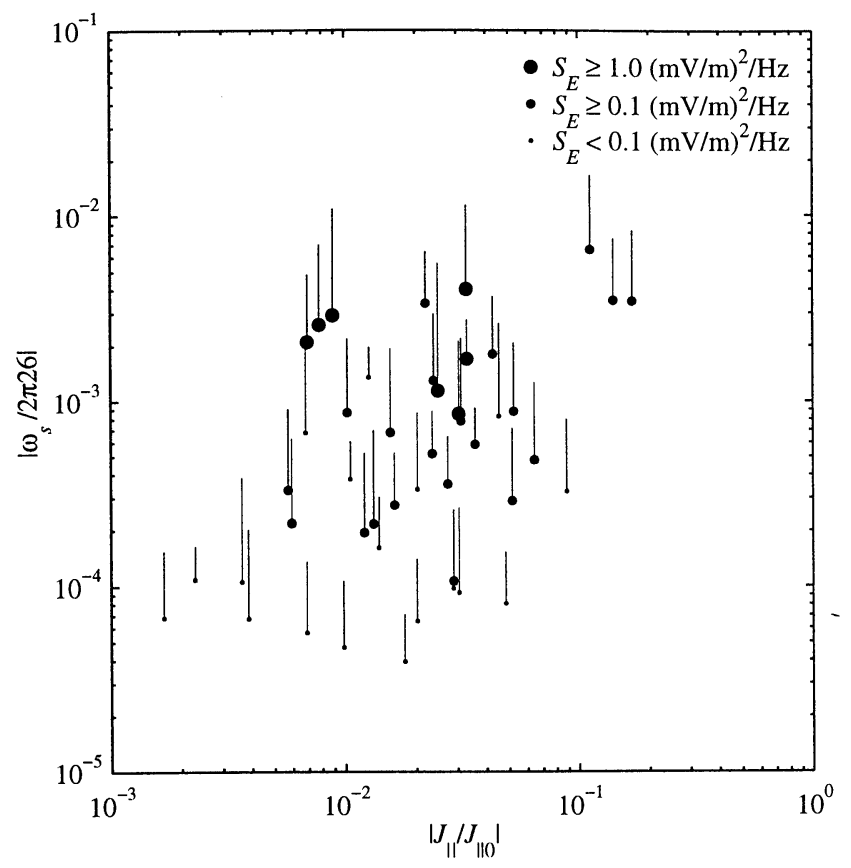

Figure 5. Normalized shear frequency during the nightside event plotted versus the absolute value of the normalized parallel magnetometer current. The data are analyzed and sorted by the power spectral density of the electric field in the same way as in Figure 4. Large values of the shear frequency are well correlated with high wave activity.

$J_{\|}$, the qualitative trend in the data is consistent with trends found theoretically and experimentally in studies of growth rate and excitation thresholds [Ganguli et al., 1988; Amatucci et al., 1994; Koepke et al., 1995, 1998a; Gavrishchaka et al., 1996, 1997].

To further investigate the importance of the observed shear for the wave generation, we need to compare the Freja data with theory and laboratory experiments using dimensionless parameters similar to the ones used here. This is done in sections 4.3 and 4.4 .

\subsection{Event 2: Nightside}

For the statistical study of a nightside event, we use $\sim 12$ min of data from the nightside magnetosphere from Freja orbit 5961 ( 0834-0846 UT, December 31, 1993). Detailed investigations show that ion energization is associated with broadband waves and also sometimes with lower-amplitude waves near the lower hybrid frequency. Broadband waves are found in the same region (but usually not exactly on the same field line) as precipitating $\mathrm{keV}$ electrons during this event. The result from the statistical study is presented in Figure 5, and the data are prepared in the same way as in Figure 4 using the same averaging and normalizing methods.

We see that the result from this nightside event is similar to the result from the statistical analysis of our dayside event. It is obvious that the spectral density is higher toward the upper part of the figure, where the shear frequency is higher, and no obvious dependence on the parallel current is seen. However, it can be noted that the overall shear frequency of the nightside event is somewhat smaller than for the dayside event.

\subsection{Scale Size Effects on the Shear}

The theory of the nonresonant instability states that the conditions for wave growth are favorable when the scale size of the transverse DC electric field structures are only a few gyroradii, although the resonant waves can exist for a much larger scale sizes. Our assumed scale size of the static electric field structures of the order of $1.4 \mathrm{~km}$ or 29 ion gyroradii (oxygen ions of energy $10 \mathrm{eV}$ ) might therefore be too large to be relevant. However, the assumed scale size corresponds to a Dopplershifted frequency of $\sim 5 \mathrm{~Hz}$ as measured by Freja. This frequency is clearly much smaller than the oxygen gyrofrequency $(26 \mathrm{~Hz})$, which makes comparisons between static structures and generated waves meaningful.

Shorter length scales of the static transverse electric fields makes the estimated sheared frequency higher. This can be seen in Figure 6, where we present one 1.5-

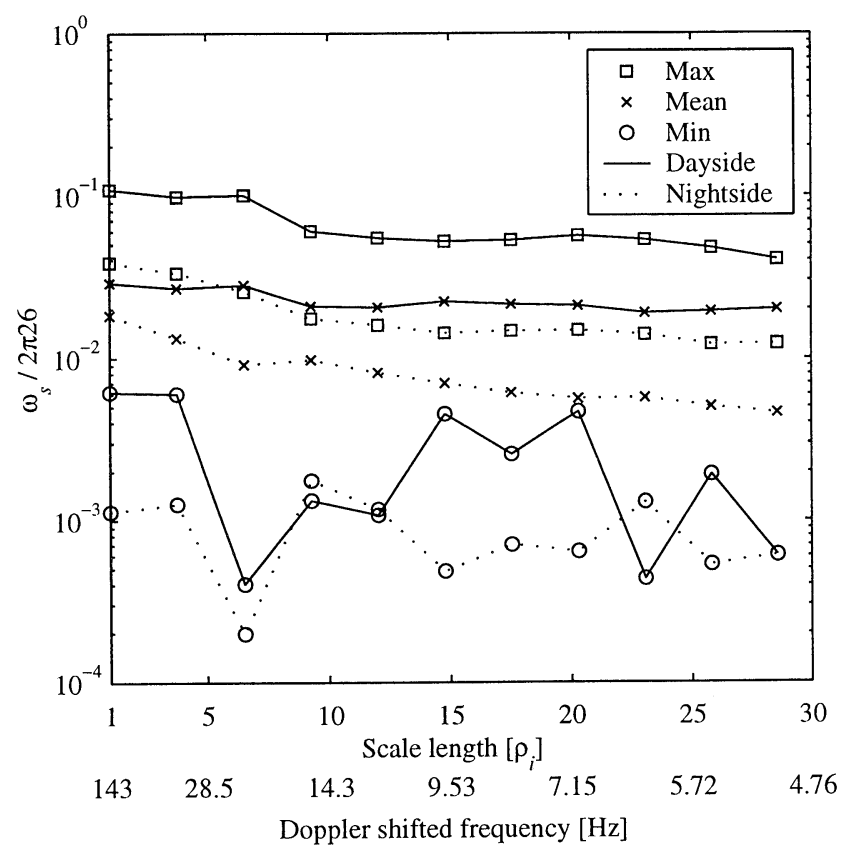

Figure 6. The normalized shear frequency as a function of the length scale of the transverse electric field assumed to be static and versus the corresponding Doppler-shifted frequency as measured by Freja in the moving satellite frame. Two 1.5-s-long time series from the dayside event (solid curves) and the nightside event (dotted curves) are used. The maximum, average, and minimum shear frequency are indicated by squares, crosses, and circles, respectively. The value of the shear frequency increases as the scale size of the static electric field structures decreases. For meaningful comparisons in our study, the Doppler-shifted frequency of the assumed static structures must be much smaller than the ion gyrofrequency $26 \mathrm{~Hz}$. 


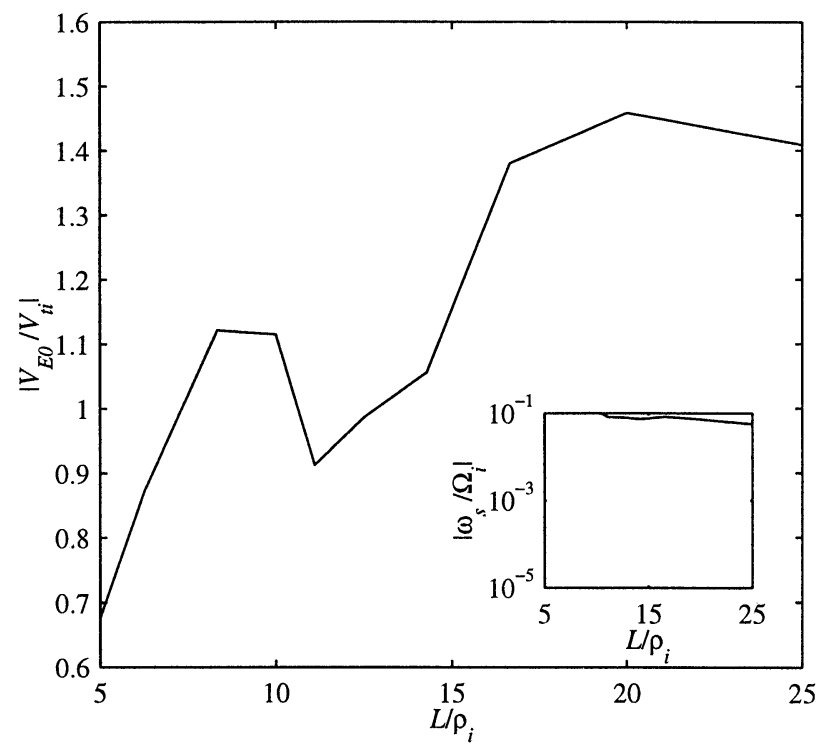

Figure 7. Critical normalized $\mathbf{E} \times \mathbf{B}$ drift versus $L / \rho_{i}$ for a typical nonresonant inhomogeneous energy density-driven (IEDD) root. A dispersion equation with a sharp-boundary velocity profile $\left[V_{E}(x)=V_{E 0}\right.$ for $|x|<L$ and $V_{E}(x)=0$ otherwise] is solved. Other parameters are $T_{i} / T_{e}=1, m_{i} / m_{e}=29,392$ (oxygen plasma), and $V_{\|}=0$. Optimization over all possible $k_{y}$ with $k_{z}=0$ is performed. Inset shows the equivalent normalized shear frequency $\omega_{s} / \Omega_{i} \approx\left(V_{E} / V_{t i}\right) /\left(L / \rho_{i}\right)$ plotted versus $L / \rho_{i}$.

s-long time series from regions of high and varying shear frequency from the dayside and the nightside event, respectively. Here we have varied the assumption of the scale size of the localized transverse electric field between 1 and 29 ion gyroradii for oxygen ions of $10 \mathrm{eV}$. The dayside event (orbit 6656) is indicated with solid lines, and the nightside event (orbit 5961) is indicated with dotted lines in Figure 6. The average values of the shear frequency during the $1.5 \mathrm{~s}$ are marked with crosses. Also, the maximum and the minimum values of the shear frequency are indicated in Figure 6 (open squares and circles, respectively).

For the scale length $29 \rho_{i}$ in Figure 6, the two 1.5-slong time series are chosen exactly to correspond to one data point in Figure 4 and one data point in Figure 5, respectively. The Doppler-shifted frequency that would be measured in the moving satellite frame if the field structures were truly static is also shown in the same figure. For a meaningful comparison between static structures possibly causing the broadband waves and the waves themselves, this Doppler-shifted frequency must be less than the wave frequency of interest ( 26 $\mathrm{Hz}$ ). However, there is no clear limit saying how much smaller the Doppler-shifted frequency must be. Therefore a wide range of frequencies around the ion gyrofrequency is shown in Figure 6. From this Figure 6 it is obvious that the value of the shear frequency increases as we focus on smaller and smaller scale sizes of the electric field.

\subsection{Theory}

To be able to investigate how important the observed shear frequency and the field-aligned current might be for the generation of the broadband waves, the results from Figures 4 and 5 need to be compared with a theoretical model. For that purpose we have numerically calculated the threshold from a linear model [Ganguli et $a l ., 1994]$. For the $\mathbf{E} \times \mathbf{B}$ drift a sharp-boundary profile $\left(V_{E}(x)=V_{E 0}\right.$ for $|x|<L$ and $V_{E}(x)=0$ otherwise) is used for convenience in the calculations, and oxygen ions are assumed for modeling space conditions. This profile is not identical to the one in Figure 1, but as discussed in section 2, the wave characteristics are not very sensitive to the sharpness of the velocity profile.

In Figure 7 the threshold for a typical nonresonant mode near the ion gyrofrequency is presented. Here the absolute value of the $\mathbf{E} \times \mathbf{B}$ drift normalized to the oxygen thermal velocity $V_{t i}$ is plotted versus the normalized scale size $L / \rho_{i}$, where $\rho_{i}$ the ion gyro radius. The ion and electron temperatures are assumed to be equal, and the field-aligned drift $V_{\|}=0$. We have optimized the growth rate over all wave numbers $k_{y}$ and used $k_{z}=0$. Since we have not attempted to model any specific velocity profile in space in detail, the details of Figure 7 are of no concern. The interesting information is the order of magnitude of the parameters and the general trend that an increased $\mathbf{E} \times \mathbf{B}$ drift is needed for instability when the scale length increases.

The nonresonant mode is not sensitive to the parallel drift $V_{\|}$of the electrons, since it is the electric field inhomogeneity that is the source of the free energy. As discussed in section 2, the wave generation is dependent on both $V_{0 E}$ and $L$ and not necessarily on the combined parameter $\omega_{s}=V_{0 E} / L$. From Figure 7 it is evident that the generation of the waves is dependent both on $V_{0 E}$ and $L$ but not necessarily on the shear frequency. The shear frequency $\omega_{s} / \Omega_{i}=\left(V_{E} / V_{t i}\right) /\left(L / \rho_{i}\right)$ normalized to the oxygen gyrofrequency $\Omega_{i}$ is plotted versus $L / \rho_{i}$ and is shown as an inset in Figure 7. To facilitate the comparison between the theoretical curve in this figure with the Freja data, the range of the shear frequency in the inset of Figure 7 is the same as in Figures 4 and 5 .

The threshold for generation of waves via the resonant instability is shown in Figure 8, where the $\mathbf{E} \times \mathbf{B}$ drift normalized to the oxygen thermal velocity $V_{t i}$ is plotted versus the parallel drift $V_{\|}$normalized to the critical drift $V_{\| 0}$ of the homogeneous CDEIC mode with the same wave number. For this case a sharp-boundary electric field profile with the scale size $L / \rho_{i}=25$ is used. The other parameters are the same as in Figure 7.

For the resonant mode the primary source of the free energy is the parallel electron drift, while the inhomogeneous electric field acts as a catalyst for the wave generation. This electric field introduces additional eigenmodes having a range of frequencies and wave numbers not possible in the CDEIC case [Gavrishchaka et al., 1996; Koepke et al., 1998b]. These eigenmodes have a 


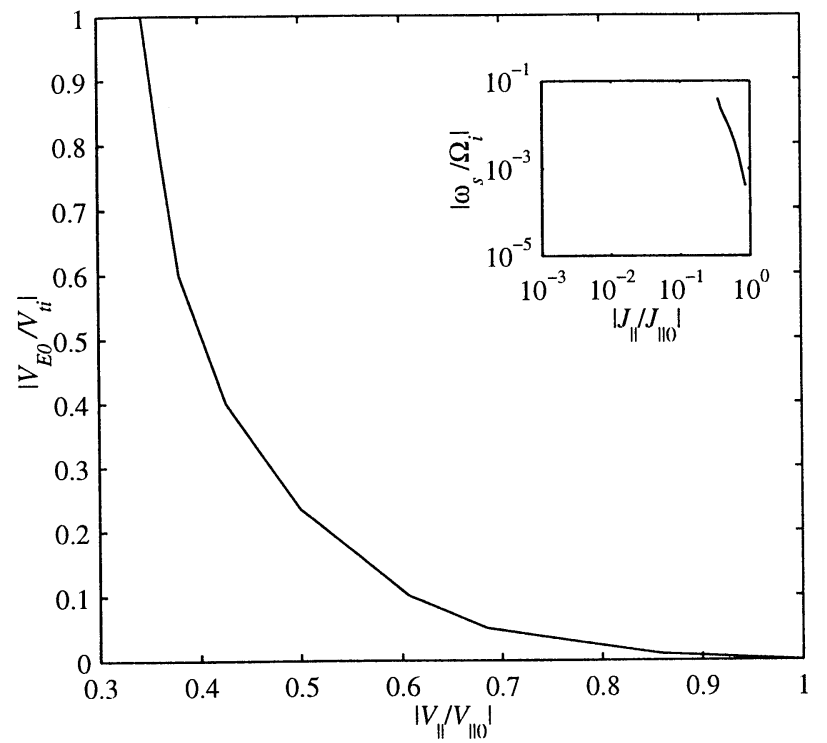

Figure 8. Critical normalized $\mathbf{E} \times \mathbf{B}$ drift versus the normalized parallel drift. Here $\left(k_{y} \rho_{i}\right)^{2}=0.12$ and optimization over $k_{z}$ is performed. $V_{\|}$is normalized to the the critical drift for the homogeneous CDEIC mode with the same wave number. A sharp-boundary transverse velocity profile with $L / \rho_{i}=25$ is used. Other parameters are the same as in Figure 8 . Inset shows the equivalent normalized shear frequency $\omega_{s} / \Omega_{i} \approx\left(V_{E} / V_{t i}\right) /\left(L / \rho_{i}\right)$ versus the parallel current normalized to the CDEIC critical current. The shear frequency (or transverse $\mathbf{E} \times \mathbf{B}$ drift) needed to generate the waves obviously decreases as the field-aligned current increases.

range of growth rates, some of which are larger than the CDEIC growth rate for the same value of the current. Consequently, the spectra of frequencies and wavenumbers are broader and the excitation threshold is smaller than for the CDEIC instability. The shift of the spectrum toward longer wavelengths has been confirmed by laboratory experiments at West Virginia University [Koepke et at., 1994; Carroll et al., 1998]. Therefore to calculate the threshold value in Figure 8 for the resonant mode, a typical value for the allowed wavelength $\left(k_{y} \rho_{i}\right)^{2}=0.12$ is chosen. From the figure we see that the threshold value of the transverse electric field decreases as the parallel drift increases, and at the point where the parallel drift is equal to the critical drift for the CDEIC mode, no inhomogeneous transverse electric field is needed for the generation of the waves.

In the top right corner of Figure 8, the absolute value of the shear frequency at the wave generation threshold plotted versus the field-aligned current is shown as an inset. The shear frequency is normalized to the oxygen gyrofrequency $\Omega_{i}$, and the current is normalized to the critical current $J_{\| 0}$ for the homogeneous CDEIC mode. The scaling of the axes of this plot is exactly the same as the scaling of Figures 4 and 5.

The two insets in Figures 7 and 8 schematically map out the excitation threshold curve for generating waves in the presence of a localized electric field using linear theory. For large parallel currents (close to the threshold current for the classical CDEIC instability) the plot in Figure 8 applies, while for small currents (much smaller than the threshold current for the classical CDEIC instability), Figure 7 is the relevant one. For large currents we note that the sheared flows needed for wave generation decrease as the field-aligned current increases. However, for small currents this dependency is not significant. From the lack of current density dependence in Figures 4 and 5, we therefore conclude that our two Freja events belong to the low current regime.

Comparing the shear frequency in Figures 4 and 5 with the excitation threshold in Figures 7 and 8 , it is obvious that the Freja data reside below the threshold curve. However, as noted in the previous sections, the value of the shear frequency is clearly dependent on the assumptions made, e.g., the scale size of the electric field structures measured by Freja. It should also be noted that the in situ measurements pertain to a possibly saturated state. Numerical simulations [Nishikawa et al., 1988, 1990] indicate that in the nonlinear saturated state, the initial electric field is much reduced in magnitude and its scale size is considerably broadened, although broadband waves are still generated. This would imply that the value of the shear frequency would be significantly smaller than its initial value. Quantifying the nonlinear shear threshold for these waves is a nontrivial task, although comparison of the in situ data with the linear threshold is, perhaps, too conservative.

\subsection{Laboratory Experiments}

The reduction of the threshold value of the fieldaligned electron drift compared to the threshold for the CDEIC instability has been confirmed experimentally [Amatucci et al., 1994, 1996; Koepke et al., 1994, 1995, 1999]. To investigate the general trends of the plasma waves due to a localized electric field in the magnetosphere, we compare our Freja data with measurements of controlled experiments of wave growth in laboratory plasmas that include a localized transverse electric field. In Figure 9, data from laboratory measurements of the wave excitation threshold for collisionless plasmas are presented. The laboratory experiments are performed in the West Virginia University Q machine [Koepke et al., 1998a] using a single ionizer on one end. This device produces a steady state, magnetized plasma column with comparable electron and ion temperatures [Rynn and D'Angelo, 1960]. Two different ion species are used, and in Figure 9, squares indicate sodium ions for the case $\left(k_{\perp} \rho_{i}\right)^{2} \approx 0.42(B=0.085 \mathrm{~T})$, while triangles and circles denote barium for the cases $\left(k_{\perp} \rho_{i}\right)^{2} \approx 0.30$ $(B=0.25 \mathrm{~T})$ and $\left(k_{\perp} \rho_{i}\right)^{2} \approx 0.68(B=0.16 \mathrm{~T})$, respectively. Moreover, the full width at half maximum of the radial field profile is $\sim 6 \mathrm{~mm}$ in all cases.

A segmented-disk end electrode produces and controls the perpendicular plasma flow and the parallel 


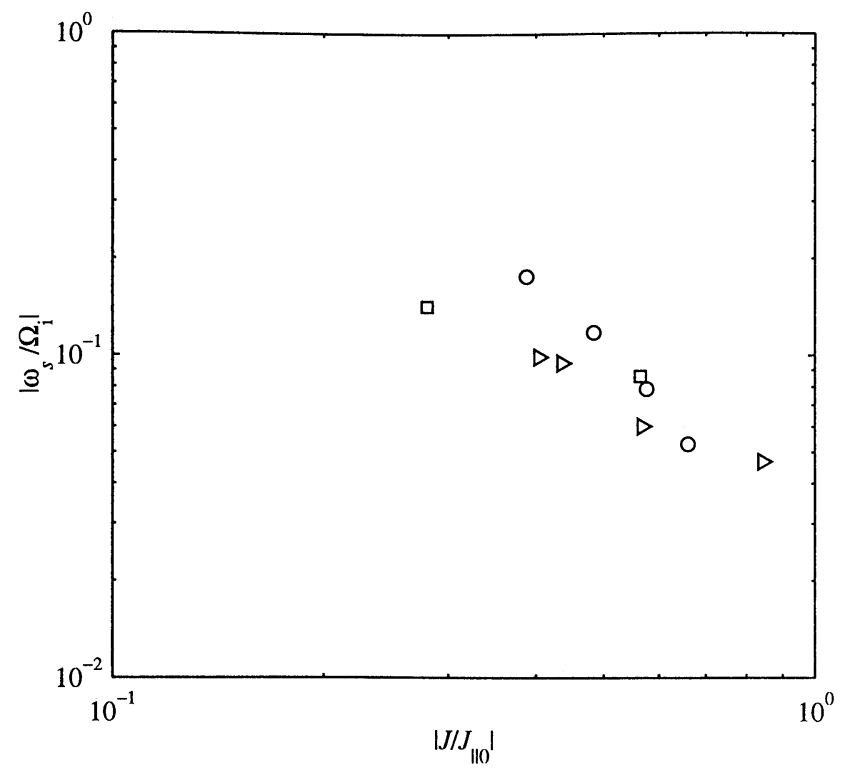

Figure 9. Threshold for wave excitation measured in the West Virginia University Q machine. The critical value of the parallel current $J_{\|}$is normalized to the critical drift of the CDEIC instability, and the peak value of the radial profile of the shear frequency $\omega_{s}$ is normalized to the ion gyrofrequency. Symbols are defined as follows: squares, sodium plasma $(B=0.085 \mathrm{~T}$, $\left.\left(k_{\perp} \rho_{i}\right)^{2} \approx 0.42\right)$; triangles, barium plasma $(B=0.25 \mathrm{~T}$, $\left.\left(k_{\perp} \rho_{i}\right)^{2} \approx 0.30\right)$; circles, barium plasma $(B=0.16 \mathrm{~T}$, $\left.\left(k_{\perp} \rho_{i}\right)^{2} \approx 0.68\right)$.

electron drift velocity [Carroll et al., 1994]. The applied voltages on the inner button segment $(r<0.40 \mathrm{~cm})$ and on the outer annular segment $(0.40 \mathrm{~cm}<r<7.5 \mathrm{~cm})$ are set with independent power supplies so that they differ by $0-5 \mathrm{~V}$, resulting in a radially localized radial electric field in the range $0-100 \mathrm{~V} / \mathrm{m}$. In this cylindrical geometry it is the radial profile of the azimuthal drift velocity that is inhomogeneous. The parallel current is adjusted, independent of the electric field profile, by varying the bias of the ionizer.

Emissive probes are used to measure radial profiles of the plasma potential, from which are obtained profiles of the transverse electric field. On the basis of the current density collected by either the end electrode or a single-sided Langmuir probe and the electron's density and charge, the parallel electron drift speed can be determined [Amatucci et al., 1994].

For a given radial electric field profile the value of the parallel electron drift speed corresponding to the instability excitation threshold is determined by raising the bias of the ionizer until evidence of the mode in the fluctuation spectrum barely exists. Then the ionizer bias is precisely adjusted to obtain a maximum value of parallel electron drift speed for which no waves are detected, i.e., the threshold value.

From the maximum of the radial electric field and the full width at half maximum of the profile, we have calculated the corresponding shear frequency. In Figure
9 the threshold for wave excitation is shown. Here the normalized shear frequency is plotted versus the parallel current needed to generate waves in the absence of a nonuniform electric field profile in the lab plasma, i.e, the critical current for the CDEIC instability. From Figure 9 it is obvious that as the field-aligned current increases to the right of Figure 9, the shear frequency needed for the wave generation decreases. These laboratory results are consistent with the theoretical predictions of Figure 8 showing that the theory is applicable to this kind of wave generation. When comparing Figure 9 to Figures 4 and 5, we see that the lab data apply to higher parallel currents than the Freja data. Furthermore, we cannot see a clear dependence on the parallel current in Figures 4 and 5. This is likely because the field-aligned current is too small to have a significant influence on the wave generation in our two events.

\section{Discussion}

It is well known that waves can be generated in inhomogeneous plasma. For example, the Kelvin-Helmholtz instability has been thoroughly investigated both experimentally [Jassby, 1972] and theoretically [Miura and Sato, 1978; Miura and Pritchett, 1982]. In this article we have focused on the instabilities due to nonuniform static electric field structures perpendicular to the geomagnetic field, and thus we have ignored the effects of inhomogeneous densities and parallel flows. The instabilities we consider depend on the magnitude of the electric field and its scale size. To keep the analysis tractable, we use the local value of the shear frequency instead of the global value in equation (1) when comparing the theoretical predictions and the laboratory data with in situ measurements from Freja. However, ongoing research suggests that there may also be other instabilities that could be driven by the local values of shear.

Without proving that the electric field signatures detected by Freja correspond to static structures in the magnetosphere, we have made the reasonable assumption that the low-frequency signatures correspond to static structures with scale lengths of a few kilometers that are Doppler shifted in the moving spacecraft frame. The signals at slightly higher frequencies are assumed to be associated with genuine time variations (i.e., waves). The assumption that all signals observed at low frequency are caused by purely static structures results in an overestimate of the importance of sheared flows for generating the waves. It is more likely that the observed signals are a mixture of Doppler-shifted static structures and genuine time-varying fields, but spacetime ambiguities and lack of wave vector measurements by Freja prevent accurate estimates of Doppler shifts.

In this article we have correlated broadband wave activity with values of the field-aligned current and the spatial inhomogeneities in the transverse electric field for several minutes of data from the Freja satellite. We have compared our findings with laboratory mea- 
surements and with theoretical predictions. However, care should be taken when comparing quantitatively the Freja data in Figures 4 and 5, the theoretical threshold curve in Figures 7 and 8, and the lab threshold curve in Figure 9, since the various figures correspond to different plasma conditions. The Freja data most probably pertain to a saturated nonlinear steady state, while the curves in Figures 7 and 8 show the linear theory excitation threshold. Also, the lab data in Figure 9 map out the threshold curve but for different ion species, i.e., barium and sodium instead of oxygen, and the boundary conditions in the laboratory device may introduce a limitation on the available wavelengths. The Freja data indicate that the average shear frequency is an order of magnitude smaller than the shear frequencies associated with the threshold curves in Figures 7-9. However, as discussed in section 4.1, the maximum shear frequency indicated by lines in Figures 4 and 5 gives a higher estimate. This shear frequency is still below the threshold. One interpretation of this discrepancy is that waves may exist in a nonlinear state of the plasma [Nishikawa et al., 1988, 1990], where, because of the presence of the waves, the marginal stability condition occurs at smaller values of the shear frequency than predicted by linear theory. It is beyond the scope of this paper to predict the marginal stability condition for the nonlinear steady state.

The theoretical model concerns a localized equilibrium electric field configuration as shown in Figure 1, but from our Freja measurements we know that such single, isolated electric field structures are not common in the ionosphere and magnetosphere (see Figures 2 and 3). Theoretical studies [Reynolds and Ganguli., 1998] and laboratory experiments [Koepke et al., 1998b] show that the existence of multiple shear layers significantly reduces the values of the shear frequency and the current density necessary for wave excitation.

The estimated shear frequency depends on the satellite orbit through the plasma flow region. An orbit perpendicular to the plasma flow (i.e., along the $x$ axis in Figure 1) will give the highest estimate of the shear. However, the exact orientation of the flow region is not known, and most orbits will give an underestimate of the shear frequency.

Our assumed scale length of the static transverse electric field (1.4 km or longer) corresponds to Freja observations of sheared plasma flows slightly below the theoretical threshold for wave generation. This scale size corresponds to a Doppler shift caused by the satellite motion to frequencies well below the $\mathrm{O}^{+}$gyrofrequency. Assuming much shorter scale lengths means that structures are Doppler shifted to frequencies near or above the wave frequency of interest, making the interpretation of the results complicated. However, decreasing the assumed scale length of the static structures (reducing the averaging; see Figure 6), we find that the shear frequency increases and approaches the threshold for wave generation.

\section{Conclusions}

We have evaluated the importance of localized electric fields in giving rise to broadband wave instabilities in the magnetosphere. Data from the Freja satellite have been used and compared with theoretical predictions and laboratory data.

There are inherent problems when comparing satellite observations with theoretical models and laboratory data. For example, because of the space and time ambiguity in the satellite data, our results depend on certain assumptions in our analysis of the velocity shear. Also, the theoretical models explicitly include some plasma flow profile parameters, the peak drift speed $V_{0 E}$, and the scale length $L$, while we estimate from the satellite data only a combination of these in the form of the local value of the shear frequency.

For large scale lengths (many ion gyroradii) we find that the observed sheared plasma flow caused by nonuniform transverse DC electric fields is below the threshold for wave generation as indicated by linear theory and collisionless laboratory experiments. However, satellite orbits that are not perpendicular to the sheared plasma flow will result in an underestimate of the shear frequency. Furthermore, at shorter length scales the observed shear approaches the threshold, but in this case the interpretation of the satellite observations is more uncertain. In addition, we note that since the observations pertain to a steady state, we should use the saturated shear value for comparison with observations. The saturated shear values are much lower than their initial values, but they are harder to quantify, since they involve numerical simulations for evaluation. Also, multiple shear layers observed in the magnetosphere, but not included in the simplest theoretical models, have a favorable influence on the wave growth.

Finally, in the regime where the parallel current is not negligible, both the theory and the laboratory results show that the critical current needed for wave generation varies as a function of the shear. Field-aligned currents estimated from Freja magnetometer data are always rather low, and the wave activity does not clearly depend on the current but, rather, requires a reasonably high shear frequency, indicating the importance of shear for wave generation. We conclude that inhomogeneous transverse electric fields might well be important for the generation of broadband waves.

More detailed theoretical models of the generation of broadband waves, for example, including other nonuniformities in the plasma than the transverse electric field, will be available in the future. This will facilitate the investigations of the importance of an inhomogeneous plasma environment for the wave generation. Together with future satellite missions resolving the space and time ambiguity in the data and laboratory experiments better adapted to the magnetospheric conditions, there is a good chance of shedding light on the processes of wave excitation in the magnetosphere. 
Acknowledgments. We gratefully acknowledge $P$ Norqvist and K. Rönnmark for useful discussions and P. Schuck for his comments. We are also grateful to the Freja principal investigators M. Boehm, L. Eliasson, B. Holback, G. Marklund, and L. Zanetti for supplying data used in this study.

Michel Blanc thanks Philippe Louarn and another referee for their assistance in evaluating this paper.

\section{References}

Aikio, A. T., L. G. Blomberg, G. T. Marklund, and M. Yamauchi, On the origin of the high-altitude electric field fluctuations in the auroral zone, J. Geophys. Res., 101, 27,157-27,170, 1996.

Amatucci, W. E., M. E. Koepke, J. J. Carroll, and T. E. Sheridan, Observation of ion-cyclotron turbulence at small values of magnetic-field-aligned current, Geophys. Res. Lett., 21, 1595-1598, 1994.

Amatucci, W. E., D. N. Walker, G. Ganguli, J. A. Antoniades, D. Duncan, J. H. Bowles, V. Gavrishchaka, and M. E. Koepke, Plasma response to strongly sheared flow, Phys. Rev. Lett., 77, 1978-1981, 1996.

André, M., P. Norqvist, L. Andersson, L. Eliasson, A. I. Eriksson, L. Blomberg, R. E. Erlandson, and J. Waldemark, Ion energization mechanisms at $1700 \mathrm{~km}$ in the auroral region, J. Geophys. Res., 103, 4199-4222, 1998.

Basu, S., S. Basu, E. Mackenzie, P. F. Fougere, W. R. Coley, N. C. Maynard, J. D. Winningham, M. Sugiura, W. B. Hanson, and W. R. Hoegy, Simultaneous density and electric field fluctuation spectra associated with velocity shears in the auroral oval, J. Geophys. Res., 93, 115-136, 1988.

Berthelier, A., J.-C. Cerisier, J.-J. Berthelier, and L. Rezeau, Low-frequency magnetic turbulence in the high-latitude topside ionosphere: Low frequency waves or field-aligned currents?, J. Atmos. Terr. Phys., 53, 333-341, 1991.

Boehm, M., et al., The TESP electron spectrometer and correlator (F7) on Freja, Space Sci. Rev., 70, 509-540, 1994.

Bonnell, J., Identification of broadband ELF waves observed during transverse ion acceleration in the auroral ionosphere, $\mathrm{PhD}$ dissertation, Dep. of Electr. Eng., Cornell Univ., Ithaca, N.Y., 1997.

Bonnell, J., P. Kintner, J. E. Wahlund, K. Lynch, and R. Arnoldy, Interferometric determination of broadband ELF wave phase velocity within a region of transverse auroral ion acceleration, Geophys. Res. Lett., 23, 3297-3300, 1996.

Carroll, J. J., M. E. Koepke, W. E. Amatucci, T. E. Sheridan, and M. J. Alport, A segmented disk electrode to produce and control parallel and transverse particle drifts in a cylindrical plasma, Rev. Sci. Instrum., 65, 2991-2995, 1994.

Carroll, J. J., M. E. Koepke, M. W. Zintl, V. Gavrishchaka, and G. Ganguli, Velocity-shear origin of low-frequency electrostatic ion-gyroresonant wave, Geophys. Res. Lett., 25, 3099-3102, 1998.

Chang, T., G. B. Crew, N. Hershkowitz, J. R. Jasperse, J. M. Retterer, and J. D. Winningham, Transverse acceleration of oxygen ions by electromagnetic ion cyclotron resonance with broad band left-hand polarized waves, Geophys. Res. Lett., 13, 636-639, 1986.

Drummond, W. E., and M. N. Rosenbluth, Anomalous diffusion arising from microinstabilities in plasma, Phys. Flu$i d s$, 5, 1507-1513, 1962.

Dubinin, E. M., P. L. Israelevich, N. S. Nikolaeva, I. Kutiev, and I. M. Podgorny, Localized auroral disturbance in the morning sector of topside ionosphere as a standing electromagnetic wave, Planet. Space Sci., 33, 597-606, 1985.

Dusenbery, P. B., and L. R. Lyons, Generation of ion-conic distribution by upgoing ionospheric electrons, J. Geophys. Res., 86, 7627-7638, 1981.

Earle, G. D., M. C. Kelley, and G. Ganguli, Large velocity shears and associated electrostatic waves and turbulence in the auroral F region, J. Geophys. Res., 94, 15,321$15,333,1989$.

Ergun, R. E., et al., FAST satellite observations of largeamplitude solitary structures, Geophys. Res. Lett., 25, 2041-2044, 1998.

Ganguli, G., and P. J. Palmadesso, Electrostatic ion instabilities in the presence of parallel currents and transverse electric fields, Geophys. Res. Lett., 15, 103-106, 1988.

Ganguli, G., Y. C. Lee, and P. Palmadesso, Electrostatic ion-cyclotron instability caused by a nonuniform electric field perpendicular to the external magnetic field, Phys. Fluids., 28, 761-763, 1985a.

Ganguli, G., P. Palmadesso, and Y. C. Lee, A new mechanism for excitation of electrostatic ion cyclotron waves and associated perpendicular ion heating, Geophys. Res. Lett., 12, 643-646, 1985b.

Ganguli, G., Y. C. Lee, and P. J. Palmadesso, Kinetic theory for electrostatic waves due to transverse velocity shears, Phys. Fluids, 31, 823-838, 1988.

Ganguli, G., M. J. Keskinen, H. Romero, R. Heelis, T. Moore, and C. Pollock, Coupling of microprocesses and macroprocesses due to velocity shear: An application to the low-altitude ionosphere, J. Geophys. Res., 99, 88738889, 1994.

Gavrishchaka, V. V., M. E. Koepke, and G. Ganguli, Dispersive properties of a magnetized plasma with a field-aligned drift and inhomogeneous transverse flow, Phys. Fluids, 3, 3091-3106, 1996.

Gavrishchaka, V. V., M. E. Koepke, and G. I. Ganguli, Ion cyclotron modes in a two-ion-component plasma with transverse-velocity shear, J. Geophys. Res., 102, 11,65311,663, 1997.

Gavrishchaka, V. V., S. B. Ganguli, and G. I. Ganguli, Electrostatic oscillations due to filamentary structures in the magnetic-field-aligned flow: The ion-acoustic branch, $J$. Geophys. Res., 104, 12,683-12,693, 1999.

Gavrishchaka V. V., G. I. Ganguli, W. A. Scales, S. P. Slinker, C. C. Chaston, J. P. McFadden, R. E. Ergun, and C. W. Carlson, Multiscale coherent structures and broadband waves due to parallel inhomogeneous flows, Phys. Rev. Lett., 85, 4285-4288, 2000.

Gurnett, D. A., R. L. Huff, J. D. Menietti, J. L. Burch, J. D. Winningham, and S. D. Shawhan, Correlated lowfrequency electric and magnetic noise along the auroral field lines, J. Geophys. Res., 89, 8971-8985, 1984.

Gustafsson, G., M. André, L. Matson, and H. Koskinen, On waves below the local proton gyrofrequency in auroral acceleration regions, J. Geophys. Res., 95, 5889-5904, 1990.

Hultqvist, B., M. André, S. P. Christon, G. Paschmann, and D. G. Sibeck, Contributions of different source and loss processes to the plasma content of the magnetosphere, Space Sci. Rev., 88, 373-382, 1999.

Jassby, D. L., Transverse velocity shear instabilities within a magnetically confined plasma, Phys. Fluids, 15, 1590$1604,1972$.

Kelley, M. C., and C. W. Carlson, Observations of intense velocity shear and associated electrostatic waves near an auroral arc, J. Geophys. Res., 82, 2343-2348, 1977.

Kindel, J. M., and C. F. Kennel, Topside current instabilities, J. Geophys. Res., 76, 3055-3078, 1971.

Kintner, P. M., J. Bonnell, R. Arnoldy, K. Lynch, C. Pollock, and T. Moore, SCIFER - Transverse ion acceleration 
and plasma waves, Geophys. Res. Lett., 23, 1873-1876, 1996.

Kintner, P. M., J. Franz, P. Schuck, and E. Klatt, Interferometric coherency determination of wavelength or what are broadband ELF waves?, J. Geophys. Res., 105, 21,237$21,250,2000$.

Kivanç, Ö., and R. A. Heelis, On relationships between horizontal velocity structure and thermal ion upwellings at high latitudes, Geophys. Res. Lett., 26, 1829-1832, 1999.

Knudsen, D. J., J. H. Clemmons, and J. E. Wahlund, Correlation between core ion energization, suprathermal electron bursts, and broadband ELF plasma waves, J. Geophys. Res., 103, 4171-4186, 1998.

Koepke, M. E., and W. E. Amatucci, Electrostatic ioncyclotron wave experiments in the WVU Q-machine, IEEE Trans. Plasma Sci., 20, 631-635, 1992.

Koepke, M. E., W. E. Amatucci, J. J. Carroll, and T. E. Sheridan, Experimental verification of the inhomogeneous energy-density driven instability, Phys. Rev. Lett., 72, 3355-3358, 1994.

Koepke, M. E., W. E. Amatucci, J. J. Carroll, V. Gavrishchaka, and G. Ganguli, Velocity-shear-induced ioncyclotron turbulence: Laboratory identification and space applications, Phys. Plasmas, 2, 2523-2531, 1995.

Koepke, M. E., J. J. Carroll, and M. W. Zintl, Excitation and propagation of electrostatic ion-cyclotron waves in plasma with structured transverse flow, Phys. Plasmas, 5, 1671-1680, 1998a.

Koepke, M. E., J. J. Carroll, M. W. Zintl, C. A. Selcher, and V. Gavrishchaka, Simultaneous observation of multiple nonlocal eigenmodes of an inhomogeneity-driven plasma instability, Phys. Rev. Lett., 80, 1441-1444, 1998b.

Koepke, M. E., J. J. Carroll, and M. W. Zintl, Laboratory simulation of broadband ELF waves in the auroral ionosphere, J. Geophys. Res., 104, 14,397-14,415, 1999.

Lühr, H., J. F. Warnecke, and M. K. A. Rother, An algorithm for estimating field-aligned currents from single spacecraft magnetic field measurements: A diagnostic tool applied to Freja satellite data, IEEE Trans. Geosci. Remote Sens., 34, 1369-1376, 1996.

Lundin, R., G. Haerendel, and S. Grahn, The Freja science mission, Space Sci. Rev., 70, 405-419, 1994.

Lysak, R. L., M. K. Hudson, and M. Temerin, Ion heating by strong electrostatic ion cyclotron turbulence, J. Geophys. Res., 85, 678-686, 1980.

Mishin, E., and M. Banaszkiewicz, On auroral ion conics and electron beams acceleration, Geophys. Res. Lett., 25, 4309-4312, 1998.

Miura, A., and P. L. Pritchett, Nonlocal stability analysis of the MHD Kelvin-Helmholz instability in a compressible plasma, J. Geophys. Res., 87, 7431-7444, 1982.

Miura, A., and T. Sato, Shear instability: Auroral arc deformation and anomalous momentum transport, J. Geophys. Res., 83, 2109-2117, 1978.

Moore, T. E., M. O. Chandler, C. J. Pollock, D. L. Reasoner, R. L. Arnoldy, B. Austin, P. M. Kintner, and J. Bonnell, Plasma heating and flow in an auroral arc, J. Geophys. Res., 101, 5279-5297, 1996.

Moore, T. E., R. Lundin, D. Alcayde, M. André, S. B. Ganguli, M. Temerin, and A. Yau, Source processes in the high-latitude ionosphere, Space Sci. Rev., 88, 7-84, 1999.

Mozer, F. S., C. W. Carlson, M. K. Hudson, R. B. Torbert, B. Parady, J. Yatteau, and M. C. Kelley, Observations of paired electrostatic shocks in the polar magnetosphere, Phys. Rev. Lett., 38, 292-295, 1977.
Nishikawa, K. I., G. Ganguli, Y. C. Lee, and P. J. Palmadesso, Simulation of ion-cyclotron-like modes in a magnetoplasma with transverse inhomogeneous electric field, Phys. Fluids, 31, 1568-1576, 1988.

Nishikawa, K. I., G. Ganguli, Y. C. Lee, and P. J. Palmadesso, Simulation of electrostatic turbulence due to sheared flows parallel and transverse to the electric field, J. Geophys. Res., 95, 1029-1038, 1990.

Norqvist, P., M. André, L. Eliasson, A. I. Eriksson, L. Blomberg, H. Lühr, and J. H. Clemmons, Ion cyclotron heating in the dayside magnetosphere, J. Geophys. Res., 101, 13,179-13,193, 1996.

Norqvist, P., M. André, and M. Tyrland, A statistical study of ion energization mechanisms in the auroral region, $J$. Geophys. Res., 103, 23,459-23,473, 1998.

Palmadesso, P. J., T. P. Coffey, S. L. Ossakow, and K. Papadopoulos, Topside ionosphere ion heating due to electrostatic ion cyclotron turbulence, Geophys. Res. Lett., 1, 105-108, 1974.

Reynolds, M. A., and G. Ganguli, Ion Bernstein waves driven by two transverse flow layers, Phys. Plasmas, 5, 2504-2512, 1998.

Rynn, N., and N. D'Angelo, Device for generating a low temperature, highly ionized cesium plasma, Rev. Sci. Instrum., 31, 1326-1333, 1960.

Stasiewicz, K., Y. Khotyaintsev, M. Berthomier, and J.-E. Wahlund, Identification of widespread turbulence of dispersive Alfvén waves, Geophys. Res. Lett., 27, 173-176, 2000.

Wahlund, J.-E., et al., Broadband ELF plasma emission during auroral energization, 1., Slow ion acoustic waves, J. Geophys. Res., 103, 4343-4375, 1998.

Waldemark, J., and P. Norqvist, In-flight calibration of satellite ion composition data using artificial intelligence methods, IRF Sci. Rep. 226, Swed. Inst. of Space Phys.,Kiruna, 1995.

Walker, D. N., W. E. Amatucci, G. Ganguli, J. A. Antoniades, J. H. Bowles, D. Duncan, V. Gavrishchaka, and M. E. Koepke, Perpendicular ion heating by velocity-sheardriven waves, Geophys. Res. Lett., 24, 1187-1190, 1997.

M. André, Swedish Institute of Space Physics, Uppsala Division, Box 537, 75121 Uppsala, Sweden. (mats.andre@irfu.se)

J. H. Clemmons, The Aerospace Corporation, P. O. Box 92957, Mail Station M2/260, Los Angeles, CA 90009. (James.H.Clemmons@aero.org)

G. Ganguli, Plasma Physics Division, Naval Research Laboratory, Washington, DC 20375. (gang@ppd.nrl.navy.mil)

V. Gavrishchaka, Science Applications International Corporation, McLean, VA 22102. (gavrish@apo.saic.com)

M. Hamrin, Theoretical Space Physics, Umeå University, 90187 Umeå, Sweden. (hamrin@space.umu.se)

N. Ivchenko, and T. Karlsson, Royal Institute of Technology, 10044 Stockholm, Sweden. (ivchenko@plasma.kth.se; tkarlsson@plasma.kth.se)

M. Koepke, and M. W. Zintl, Physics Department, West Virginia University, Morgantown, WV 26506. (mkoepke@wvu.edu)

(Received October 9, 2000; revised November 22, 2000; accepted December 13, 2000.) 